\title{
Research on Large Deflection Deformation Reconstruction of Elastic Thin Plate Based on Strain Monitoring
}

\author{
Wenrui Wanga, ${ }^{\mathrm{a}}$, Yu Lu ${ }^{\mathrm{a},}$, , Dezong Zhao ${ }^{\mathrm{b},{ }^{*} \text {, Jiaming Zhang }}{ }^{\mathrm{a}}$, Xuesong Bai ${ }^{\mathrm{c}}$ \\ ('School of Mechanical Engineering, University of Science and Technology Beijing, Beijing, 100083, China) \\ ('Department of Aeronautical and Automotive Engineering, Loughborough University, Loughborough, LE11, United Kingdom) \\ ('National Center for Space Science, Chinese Academy of Sciences, Beijing, 100083, China) \\ *Corr. Author: Wenrui Wang, Email: gmbitwrw@ustb.edu.cn \\ Yu Lu, Email: cdks2012@163.com \\ Dezong Zhao, Email: d.zhao@lboro.ac.uk
}

\begin{abstract}
In this paper, a new deformation measurement method based on strain monitoring is proposed. Aiming at large deflection deformation of the four-sided fixed elastic thin plate structure, an algorithm for reconstructing deformation field based on surface strain monitoring is developed. The reconstruction method theoretically analyzes the thin elastic plate according to the large deflection deformation hypothesis and elastic mechanics. The experimental platform for strain monitoring of thin plate and several different loading conditions was established. Based on the discrete point strain data obtained by experiments, the strain field is reconstructed to obtain detailed strain information. Based on the analytic solution of deformation and strain information, the deformation curves of thin plates under different loads are obtained. The experimental results show that the average error of reconstruction of the elastic thin plate structure is $4.9 \%$, and the reconstruction results accurately reflect the deformation field of the structure. I still have the feeling that the contributions are not clear. The abstract contains mainly statements rather than innovations. Key words: elastic thin plate; large deflection deformation; strain monitoring; deformation reconstruction
\end{abstract}

\section{Introduction}

Elastic thin plates are widely used in engineering structures, particularly in satellite structures. In satellite structures, the slight deformation on mounting board will affect the control accuracy of the entire engineering structure, and the deformation of the thin plate structure has become a potential danger. There are many methods to measure structural deformation. Can you provide references here? For example, non-contact measurement of structures by optical image method; The structural deformation is measured directly by displacement sensor. But in practical application, especially in aerospace field, there is often not suitable space to install deformation measuring equipment. In this paper, a method of reconstructing deformation based on measuring strain is proposed to realize structural deformation measurement. This method obtains strain information through measurement and realizes structural deformation reconstruction based on deformation reconstruction algorithm. It solves the problem that the existing measurement methods are difficult to realize engineering application due to the spatial constraints.

The deformation of a thin elastic plate is different from a beam structure in terms of diverse constrain form and complex force analysis of the unit. The thin elastic plate has the following deformation modes under the condition of lateral load.

(1) When the maximum deformation is less than $1 / 5$ of the plate thickness, the thin plate suffers to small deflection deformation. In this case, the deformation of the thin elastic plate is caused by bending solely. The strain distribution is antisymmetric with respect to the mid-plane. Since the mid-plane does not stretch, the surface strain values at a given location are only different in signs.

(2) When the maximum deformation is more than $1 / 5$ of the plate thickness, the thin plate suffers to large deflection deformation. In this case, the deformation of the thin elastic plate is caused by stretching and bending. The strain distribution is not symmetrical with respect to the mid-plane. Any reference to support the technical statement?

The thinner the plate, the easier it is to produce large deflection deformation. It is assumed that the minimum edge length of the plate is $b$ and the thickness of the plate is $\delta$. When $\frac{\delta}{b}>\left(\frac{1}{8} \sim \frac{1}{5}\right)$, the 
plate belongs to thick plate, and the deformation is mainly caused by bending. When $\left(\frac{1}{100} \sim \frac{1}{80}\right)<$ $\frac{\delta}{b}<\left(\frac{1}{8} \sim \frac{1}{5}\right)$, the plate belongs to thin plate. According to the deflection of thin plate, the deformation can be divided into small deflection and large deflection. Deformation in small deflection is mainly caused by bending, while deformation in large deflection is caused by bending and stretching. When $\frac{\delta}{b}<\left(\frac{1}{100} \sim \frac{1}{80}\right)$, the plate belongs to a large deflection thin plate, and the deformation is mainly large deflection [1].

Because thin plates are prone to large deflection deformation and the harm caused by large deflection deformation is greater than small deflection deformation. This paper mainly studies the thin plate which may has large deflection deformation. Therefore, the thickness range studied in this paper is $\frac{\delta}{b}<\left(\frac{1}{8} \sim \frac{1}{5}\right)$.

With the development of structural deformation monitoring, deformation field reconstruction algorithm based on strain monitoring has attracted wide interest. The NASA Langley Research Center investigated the inverse problem of the three-dimensional displacement of the plate-shell structure, and the inverse finite element method (iFEM) is proposed based on the first-order shear deformation theory [2-4]. A powerful four-node quadrilat-eral inverse-shell element (iQS4) is introduced, which is used to monitor the displacement and stress of structures combining the iFEM algorithm in ocean engineering [5-6]. In addition, the deformation and stress of multilayer composites and sandwich structures are monitored according to the iFEM algorithm [7-8]. A new inverseshell element i3-RZT is proposed and it improved the applicability of iFEM algorithm to composite structures [9-10]. Kim et al. (2011) placed the FBG strain sensor on the surface of the blade and monitor the dynamic deformation of the blade structure combining the modal transformation algorithm according to the experimentally measured strain. The FBG strain sensing system is built (This is a short sentence. Can you explain more? What is the significance of the built system?). The modal transformation algorithm was applied to the high-precision displacement reconstruction of the structure with vibration mode based on the experimentally measured strain [11]. The accuracy and efficiency of the modal transformation algorithm are affected by the arrangement of sensor on the structure. The factors that degrade the optimal placement of the sensors will reduce the calculation accuracy of the algorithm [12]. William L. Ko et al. (2012) transformed the wing structure deformation problem into a summation problem, which is based on classical assumptions of material mechanics and beam structure deformation relationships [13]. Nicolas (2013) obtained the out-of-plane loads and the wing shape at various load levels using NASA-developed real-time load and displacement algorithms based on the strain information [14-15]. A new deformation shape-sensing methodology is investigated for real-time condition assessment, control, and health monitoring of flexible lightweight aerospace structures. The methodology implements fiber is based an optic methods and tools sensors to obtain strain measurements from the target structure and to estimate the corresponding displacement field (This sentence is too long. Divide it into two sentences.)[16-18]. Yi et al. (2012) converted the strain information into the surface curvature information of the solar panel structure and realize the real-time structure deformation reconstruction based on the surface reconstruction algorithm (too long sentence) [19]. However, this algorithm is not suitable for large deflection deformation which more often occurs, and will cause great errors. Meng et al. (2012) derived the bending deflection function based on the strain information, which was used to solve the deformation of the satellite antenna according to the small deflection theory of thin plate [20]. Since the satellite antenna structure is fixed on one side, the method is suitable to the deformation reconstruction of the cantilever beam structure. Furthermore, it is not applicable to large deflection deformation problems. Liu et al. (2016) proposed a new threesegment beam model with local flexibilities at crack tips which is developed. The three-segment beam model is used to investigate the effects of local flexibilities at crack tips on the first three natural frequencies and mode shapes of the cracked cantilever beam (too long sentence) [21].

The above methods are only applicable to the structures with specific constraints. The research of large deflection of the thin elastic plates with four edges fixed is little. The large deflection deformation of thin plate is a nonlinear problem, which is more difficult to solve. Therefore, the above reconstruction method is far from satisfying the engineering measurement requirements. This paper uses the methods of theoretical analysis, solution, experimental monitoring and experimental verification to solve the large deflection deformation of elastic thin plates This sentence does not provide any useful information. The deformation field reconstruction method based on strain 
monitoring is delivered by deducing the strain-deformation expression and implementing experiment verification. Compared with the existing research, large deflection deformation reconstruction based on strain monitoring can be realized. This method will provide an important (I dislike the word 'important') basis for thin plate deformation measurement widely used in aerospace field.

\section{Theoretical analysis of elastic thin plates with large deflection deformation}

\subsection{Relation between strain and deformation}

The object being investigated in this paper is an elastic thin plate with four edges fixed and loaded in the middle, and the size of the subjects is $1000 \times 1000 \times 3 \mathrm{~mm}$. As the installation board of satellite components, its deformation has an important impact on satellite performance. The strain distribution of a micro-body of an elastic thin plate has shown in Fig.1(a). On the basis of the small deflection deformation theory based on the Kirchhoff hypothesis, the following four hypotheses are proposed [22].

(1) Straight line hypothesis. After bending deformation and perpendicular to the curved midplane, a straight line perpendicular to the midplane of the plate before deformation is still a straight line. It is shown that the shear strain is 0 on the plane parallel to the middle-surface, namely: $\gamma_{z x}=0, \gamma_{y z}=0$.

(2) Thickness constant assumption. The deflection of each point in the board is only a function of $x, y$ and has nothing to do with $z$, namely: $w=w(x, y)$.

(3) The normal stress in the middle plane is much smaller than the other stress component assumptions. The layers are parallel to the middle surface and are not pressed/stretched against each other. The normal stress $\sigma_{z}$ along the $z$ direction is negligible.

(4) Tensile/compressive strain exists in the middle-surface hypothesis. At this time, the midsurface tensile/compression strain can be ignored.

When the thin plate belongs to (what does 'belongs to' mean?) large deflection deformation, the mid-surface tension/compression strain should be considered. So hypothesis (4) no longer holds.

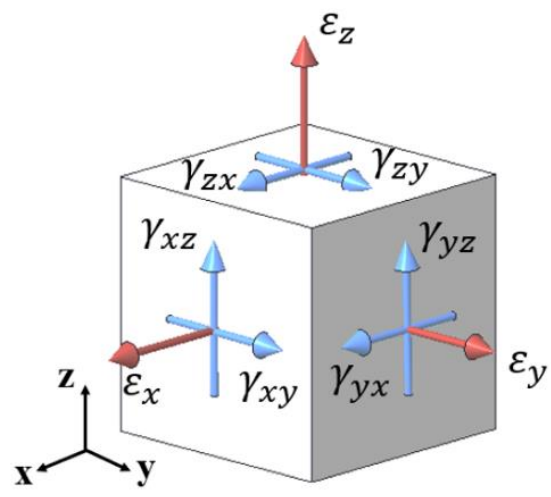

(a)

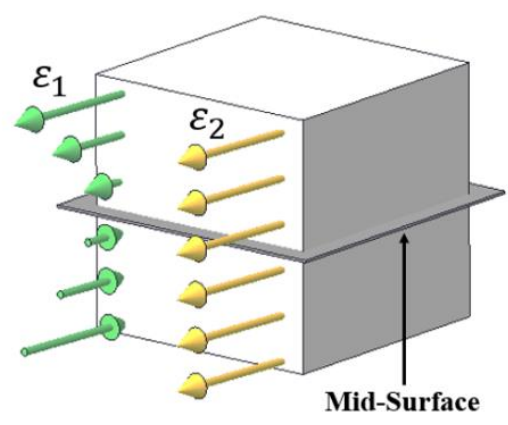

(b)

Fig.1 Strain distribution of a micro-body in thin plate

As shown in Fig.1(b), positive strain distribution diagram of a micro-body in the x-direction of a thin plate (There is no verb in the sentence!). $\varepsilon_{1}$ is the strain generated by the bending stress; $\varepsilon_{2}$ is the mid-surface tensile/compressive strain generated by membrane stress. It can be seen that $\varepsilon_{x}=$ $\varepsilon_{1}+\varepsilon_{2}$ is held under large deflection. However, small deflection deformation does not exist in $\varepsilon_{2}$.

As shown in Eq. (1), The geometric equation (Cauxi equation) is established by taking any micro-element in the thin plate as the object [23]. 


$$
\left\{\begin{array}{c}
\varepsilon_{x}=\frac{\partial u}{\partial x} \\
\varepsilon_{y}=\frac{\partial v}{\partial y} \\
\varepsilon_{z}=\frac{\partial w}{\partial z} \\
\gamma_{x y}=\frac{\partial v}{\partial x}+\frac{\partial u}{\partial y} \\
\gamma_{y z}=\frac{\partial w}{\partial y}+\frac{\partial v}{\partial z} \\
\gamma_{z x}=\frac{\partial u}{\partial z}+\frac{\partial w}{\partial x}
\end{array}\right.
$$

where $\varepsilon_{x} \varepsilon_{y}$ and $\varepsilon_{z}$ are the positive strain in the $x y$ and $z$ direction; (You should not start a new line) $\gamma_{x y} \gamma_{y z}$ and $\gamma_{z x}$ are the Shear strain of the $x y y z$ and $z x$ plane; (You should not start a new line)

$u v$ and $w$ are the displacement in the $x y$ and $z$ direction, $\mathrm{mm}$.

According to the straight line hypothesis, $\gamma_{z x}=0$ and $\gamma_{y z}=0$ are both held. Substituting them into Eq. (1) then the following equation is held.

$$
\left\{\begin{array}{l}
\frac{\partial u}{\partial z}+\frac{\partial w}{\partial x}=0 \\
\frac{\partial w}{\partial y}+\frac{\partial v}{\partial z}=0
\end{array}\right.
$$

Simplification of Eq. (2) yields Eq. (3):

$$
\left\{\begin{array}{l}
\frac{\partial u}{\partial z}=-\frac{\partial w}{\partial x} \\
\frac{\partial v}{\partial z}=-\frac{\partial w}{\partial y}
\end{array}\right.
$$

Then integrate the both sides of Eq. (3). There is a telescopic deformation in the middle surface, i.e., $(u)_{z=0}=u_{2}(v)_{z=0}=v_{2}$, where $u_{2}$ and $v_{2}$ are the mid-plane direction $x$ and $y$ displacement. Therefore, the integral constants are $u_{2}$ and $v_{2}$. Eq. (4) is obtained by integrating the both sides of Eq. (3).

$$
\left\{\begin{array}{l}
u=u_{2}-\frac{\partial w}{\partial x} \cdot z \\
v=v_{2}-\frac{\partial w}{\partial y} \cdot z
\end{array}\right.
$$

Deriving equation Eq. (4) for $x$ yields Eq. (5):

$$
\left\{\begin{array}{l}
\frac{\partial u}{\partial x}=\frac{\partial u_{2}}{\partial x}-\frac{\partial^{2} w}{\partial x^{2}} \cdot z \\
\frac{\partial v}{\partial y}=\frac{\partial v_{2}}{\partial x}-\frac{\partial^{2} w}{\partial y^{2}} \cdot z
\end{array}\right.
$$

Substituting the first two sub-equations of Eq. (1) with in Eq. (5) yields Eq. (6),

$$
\left\{\begin{array}{l}
\varepsilon_{x}=\varepsilon_{2 x}-\frac{\partial^{2} w}{\partial x^{2}} \cdot z \\
\varepsilon_{y}=\varepsilon_{2 y}-\frac{\partial^{2} w}{\partial y^{2}} \cdot z
\end{array}\right.
$$

where, $\varepsilon_{2 x}$ - Normal strain in the middle direction $x$;

$\varepsilon_{2 y}$ - Normal strain in the middle direction $y$.

Eq. (6) describes the relationship between strain and deformation of a flexible thin plate at a certain point under large deflection.

\subsection{Solution of the deformation base on strain}

The input form of the strain information needs to be clarified before solving the deformation. Determine the steps to achieve the solution based on the strain monitoring experimental method 
(how did you determine? This sentence does not provide any information.). Analytic solutions are provided for the modified field reconstruction algorithm.

In the strain monitoring experimental, the sensor is arranged on the surface of the mounting plate according to a certain regularity. As shown in Fig.2, The strain obtained by the monitoring will obtain more detailed strain information through the interpolation algorithm. The strain information is evenly distributed on the bottom surface of the entire elastic thin plate. The direction $x$ uniformly distributes the M sets of strain points with a spacing of $a$. The direction $y$ uniformly distributes the $\mathrm{N}$ sets of strain points with a spacing of $b$.

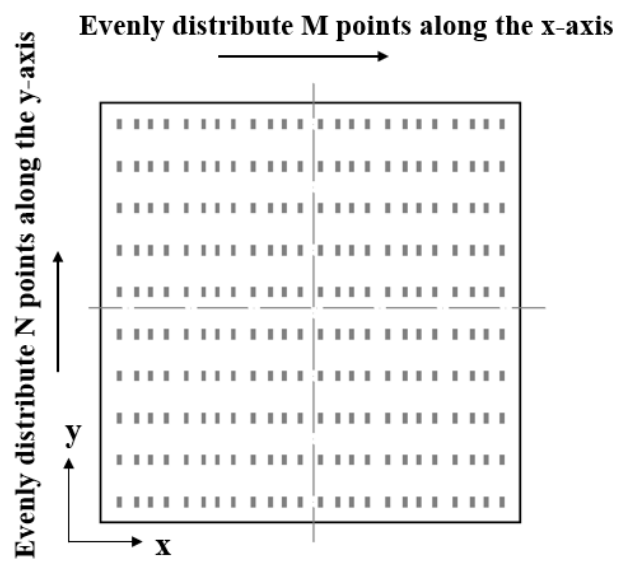

Fig.2 Strain point distribution chart on the surface of thin plates

The strain information includes the coordinates and strain values of the various strain points and will be used as the input to solve the deformation equation. The strain information are expressed in the form of a matrix, and the matrix containing the strain information is denoted as $[\varepsilon]_{N \times M}$.

According to the relationship between strain and deformation Eq. (6), the strain information obtained along the $x$-axis or along the $y$-axis can be used to solve the deformation. In this work, the direction along the $x$-axis is selected as the investigate direction. Therefore, the strain in the direction $x$ can be expressed as a matrix in Eq. (7).

$$
\left[\varepsilon_{x}\right]_{N \times M}=\left[\begin{array}{cccc}
\varepsilon_{x 11} & \varepsilon_{x 12} & \ldots & \varepsilon_{x 1 M} \\
\varepsilon_{x 21} & \varepsilon_{x 22} & \ldots & \varepsilon_{x 2 M} \\
\ldots & \ldots & \ldots & \ldots \\
\varepsilon_{x N 1} & \varepsilon_{x N 2} & \ldots & \varepsilon_{x N M}
\end{array}\right]
$$

When solving the relational equation of strain and deformation, firstly the mesh is divided into $\mathrm{N}$ parallel lines. The deformation is then reconstructed into $\mathrm{N}$ lines separately. Afterwards, the deformation of $\mathrm{N}$ straight lines will be fitted into a deformed surface.

(What do you want to do in the paragraph? A sentence is lacking at the beginning) First, the $\mathrm{M}$ group strain points $\left(\left[\varepsilon_{x n 1} \varepsilon_{x n 2} \varepsilon_{x n 3} \ldots \varepsilon_{x n M}\right] \mathrm{n}=1,2,3 \ldots \mathrm{N}\right)$ on the $\mathrm{n}^{\text {th }}$ line are taken as the object, solving the deformation curves of $\mathrm{n}^{\text {th }}$ line. Then the deformation curves of other line are solved using the same method. Finally, all the deformation curves are fitted and get the entire deformed surface is obtained.

The bottom surface of the thin plates has the coordinates $z=-t / 2$. According to the solution method proposed in the previous paragraph, the strain in the direction $x$ is taken as the research object and the expression of strain and deformation relation is simplified from Eq. (6) to Eq. (8):

$$
\frac{\partial^{2} w(x)}{\partial x^{2}}=-\frac{2}{t}\left(-\varepsilon_{x n m}^{-}(x)+\varepsilon_{2 x n m}(x)\right)
$$

where, $\varepsilon_{x n m}^{-}(x)$ - The bottom surface strain expression of the $\mathrm{n}^{\text {th }}$ curve;

$t$ - Thickness of thin plate, $\mathrm{mm}$.

To obtain the mid-surface tensile/compressive strain, it is necessary to use the strain of the top and bottom surfaces. As shown in Fig.3, the top surface strain is recorded as $[\varepsilon]_{N \times M}^{+}$, and the bottom surface is recorded as $[\varepsilon]_{N \times M}^{-}$. 


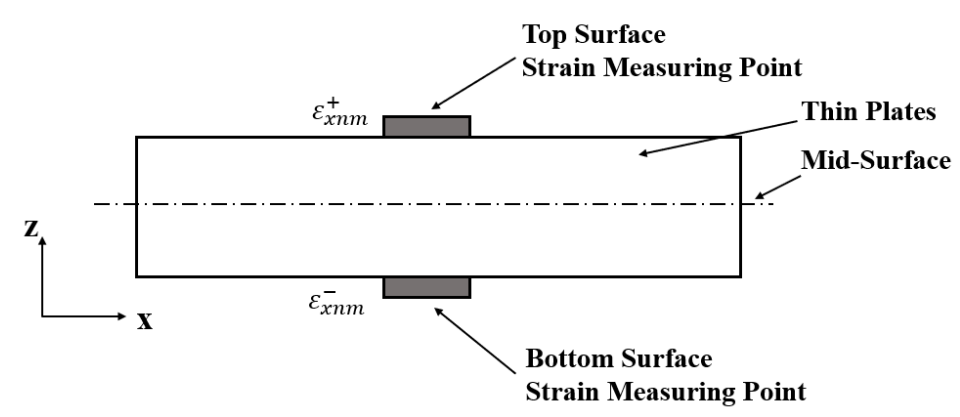

Fig.3 The Sectional view for thin plates

According to the strain information of the top and bottom surfaces of the same position, the tensile/compressive strain at the mid-surface position can be obtained as $\varepsilon_{2 x n m}$, and its expression is shown as Eq. (9).

$$
\varepsilon_{2 x n m}=\frac{\varepsilon_{x n m}^{+}+\varepsilon_{x n m}^{-}}{2}
$$

According to the solution method, Eq. (9) is reformatted as Eq. (10).

$$
\varepsilon_{2 x n m}(x)=\frac{\varepsilon_{x n m}^{+}(x)+\varepsilon_{x n m}^{-}(x)}{2}
$$

where, $\varepsilon_{x n m}^{+}(x)$ is the top surface strain expression of the $\mathrm{n}^{\text {th }}$ curve.

(do not need to start a new paragraph) Based on the expression of the mid-face tensile/compressive strain, the relationship between strain and deformation is expressed as Eq. (11):

$$
\frac{\partial^{2} w(x)}{\partial x^{2}}=\frac{2 \varepsilon_{x n m}^{-}(x)}{t}-\frac{\left(\varepsilon_{x n m}^{+}(x)+\varepsilon_{x n m}^{-}(x)\right)}{t}
$$

(do not need to start a new paragraph) The first-order integral and second-order integral on both sides of Eq. (11) is solved, and Eq. (12) and Eq. (13) are obtained:

$$
\begin{gathered}
\frac{\partial w(x)}{\partial x}=\int \frac{\varepsilon_{x n m}^{-}(x)-\varepsilon_{x n m}^{+}(x)}{t} d x+a \\
w(x)=\iint \frac{\varepsilon_{x n m}^{-}(x)-\varepsilon_{x n m}^{+}(x)}{t} d x d x+a x+b
\end{gathered}
$$

The constraint end is defined as the initial position, and the deformation value is $w(0)=0$. Due to the four-sided constraint, the initial position has $\partial w(0) / \partial x(0)=0$. In summary, $a$ and $b$ in Eq. (13) are both 0. Thus, Eq. (13) is reduced to Eq. (14).

$$
w(x)=\iint \frac{\varepsilon_{x n m}^{-}(x)-\varepsilon_{x n m}^{+}(x)}{t} d x d x
$$

(do not need to start a new paragraph) Eq. (14) is an analytic expression for the deformation of a single curve. By solving the second integral of Eq. (14), the curve deformation $w(x)$ is obtained (how it looks like?).

\section{Strain monitoring experiment and strain field reconstruction 3.1 Experimental study on strain monitoring}

The model of the strain monitoring experimental platform is shown in Fig.4. The thin plates are fixed to the fixing frame by screw. The Weight is placed under the thin plate, and a static load is applied to the thin plate by the weight. The weights used in this paper are divided into $50 \mathrm{~N}$ and $100 \mathrm{~N}$. In addition, the deformation sensor is responsible for testing the deformation of the thin plates. 


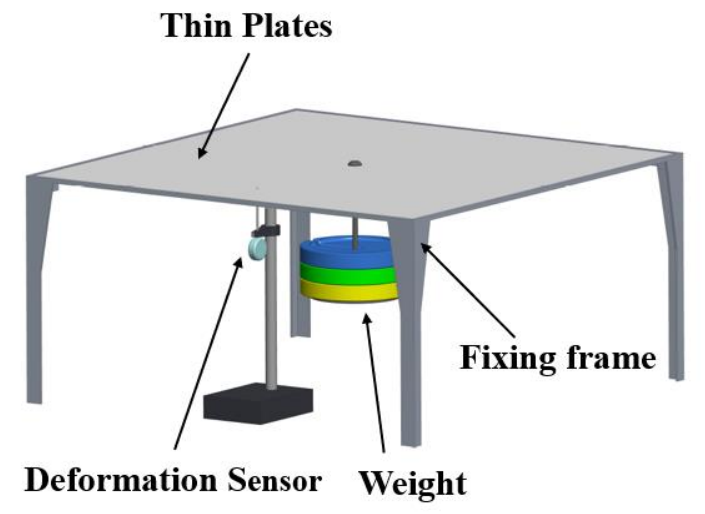

Fig.4 Model of the strain monitoring experimental platform

The photos of the strain monitoring experimental platform are illustrated in Fig.5. The strain sensor is arranged on the surface of the thin plate according to a certain regularity. The strain sensor is connected to the collector through the channel line, and the collector is connected to the PC. Finally, the strain data is displayed and recorded by a PC. The experimental instrument parameters are listed in Tab.1.

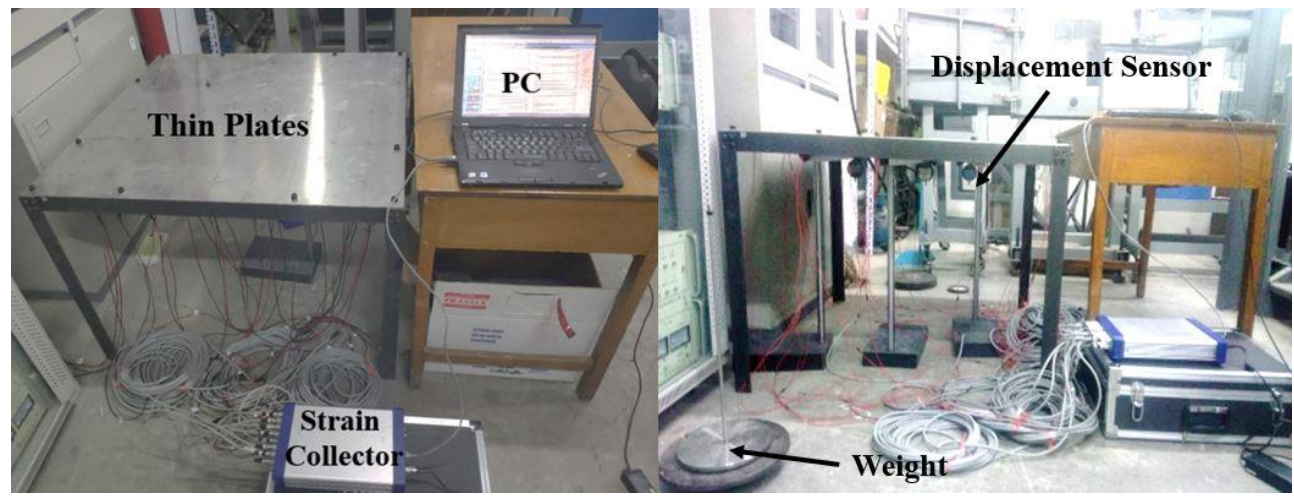

Fig.5 Photo of the strain monitoring experimental platform

Tab. 1 experimental instrument parameters

\begin{tabular}{cccc}
\hline Equipment Name & Model & Features & Precision \\
\hline Strain Sensor & BX120-5AA & Measuring strain & $1 \mu \varepsilon$ \\
Strain Collector & INV3062S/V & Collecting strain & $1 \mu \varepsilon$ \\
Displacement Sensor & SYA1704569 & Measuring displacement & $0.01 \mathrm{~mm}$ \\
Weight & - & load & $50 \mathrm{mg}$ \\
\hline
\end{tabular}

To verify the accuracy of the deformation field reconstruction through experiments, three deformation points were selected on the surface of the thin plate for deformation monitoring. As shown in Fig.6(a), the three points A $(300,700)$, B $(300,500)$ and C $(500,500)$ are used as deformation monitoring points. When the experimental platform is built, the displacement sensor will be installed under these points. The deformation obtained by the displacement sensor monitoring will be used to verify the accuracy of the reconstruction algorithm.

According to different loading positions, the strain monitoring experiment are implemented in two groups. The first group applies a load to the center point position and the second group applies a load at an arbitrarily position. As shown in Fig.6(b), Q1 $(500,500)$ is the loading position when the center point is loaded, and Q2 $(300,700)$ is the loading position when the arbitrarily point is loaded. 


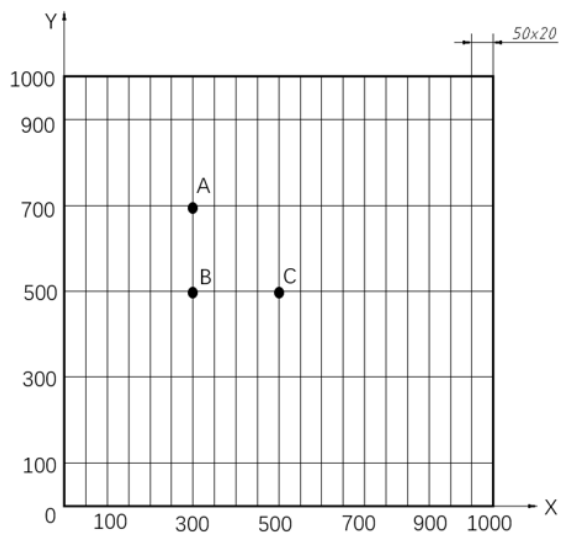

(a)

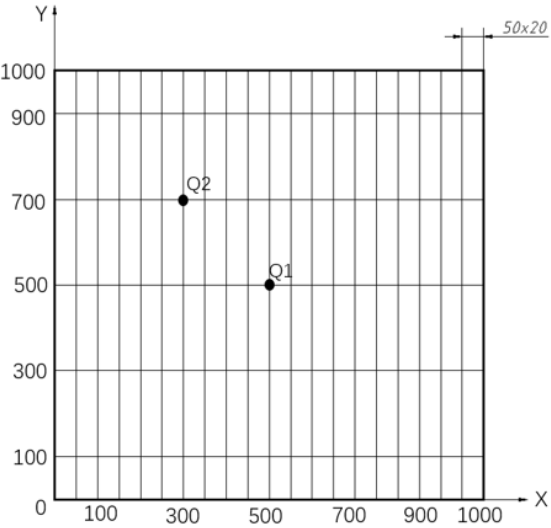

(b)

Fig.6 The chart of displacement measuring point and loading point

As shown in Tab.2, The experiment was divided into 2 groups according to the loading point position, and each loading point was tested under 4 different load conditions from $100 \mathrm{~N}$ to $250 \mathrm{~N}$. The loading from $100 \mathrm{~N}$ to $250 \mathrm{~N}$ can be realized by adding the weights in various ways.

Tab.2 Experimental loading conditions

\begin{tabular}{cccccc}
\hline $\begin{array}{c}\text { Loading } \\
\text { point }\end{array}$ & $\begin{array}{c}\text { Loading } \\
\text { position }\end{array}$ & $\begin{array}{c}\text { Load } \\
\text { condition 1 }\end{array}$ & $\begin{array}{c}\text { Load } \\
\text { condition 2 }\end{array}$ & $\begin{array}{c}\text { Load } \\
\text { condition 3 }\end{array}$ & $\begin{array}{c}\text { Load } \\
\text { condition 4 }\end{array}$ \\
\hline Q1 & $(500,500)$ & $100 \mathrm{~N}$ & $150 \mathrm{~N}$ & $200 \mathrm{~N}$ & $250 \mathrm{~N}$ \\
& & & & & \\
Q2 & $(300,700)$ & $100 \mathrm{~N}$ & $150 \mathrm{~N}$ & $200 \mathrm{~N}$ & $250 \mathrm{~N}$ \\
\hline
\end{tabular}

Based on the experimental setting up and the experimental grouping, the strain sensor layout scheme is designed (how it looks like?). The goal of the layout is to select as few sensors as possible to obtain complete thin plate surface strain information. After performing a plurality of tests, the final layout scheme obtained is shown in Fig.7 and Fig.8. Fig.7 and Fig.8 are the monitoring point layout diagrams of the center point load and the arbitrary point load strain monitoring experiment, respectively. Fig.7(a) and Fig.8(a) are the distribution diagrams of the top surface strain sensors of the elastic thin plate, and Fig.7(b) and Fig.8(b) are the distribution diagrams of the strain sensors on the bottom surface of the elastic thin plate.

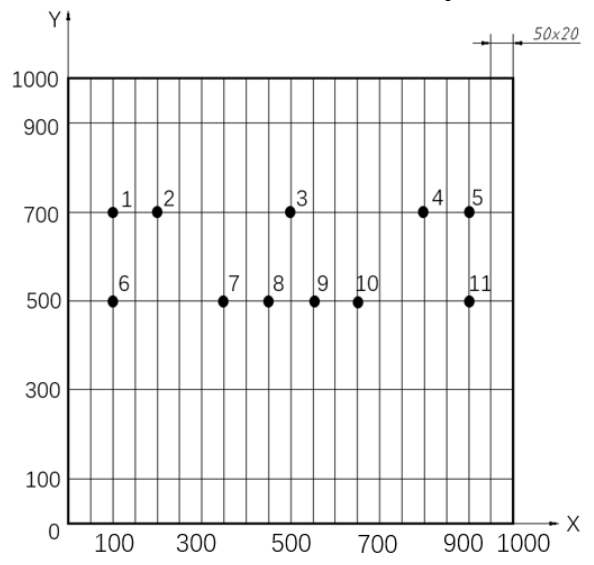

(a)

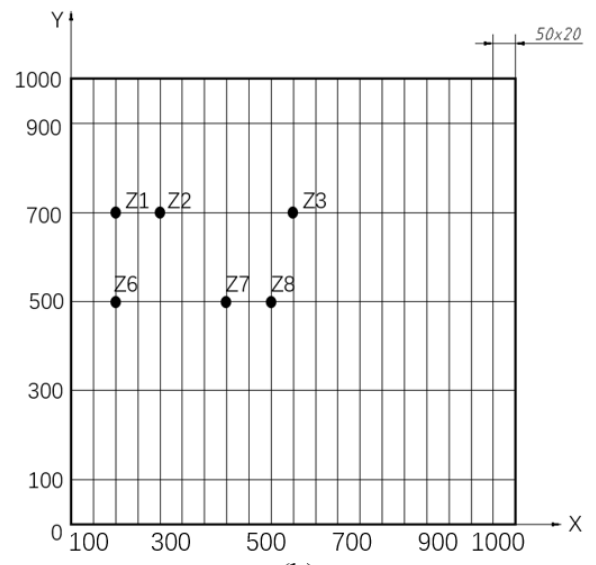

(b)

Fig.7 Strain point distribution chart of center point load 


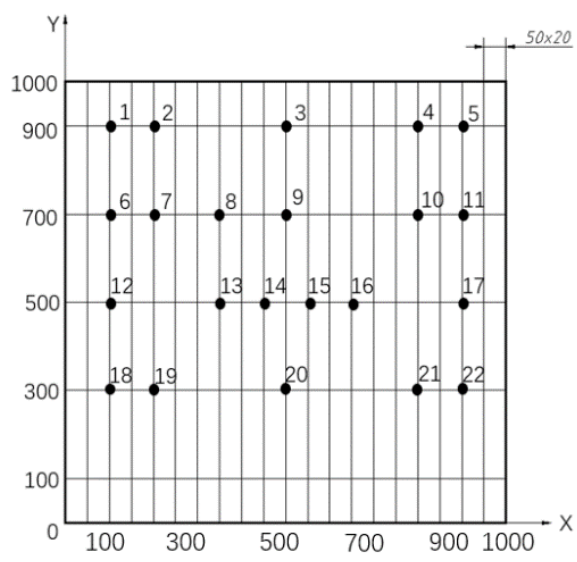

(a)

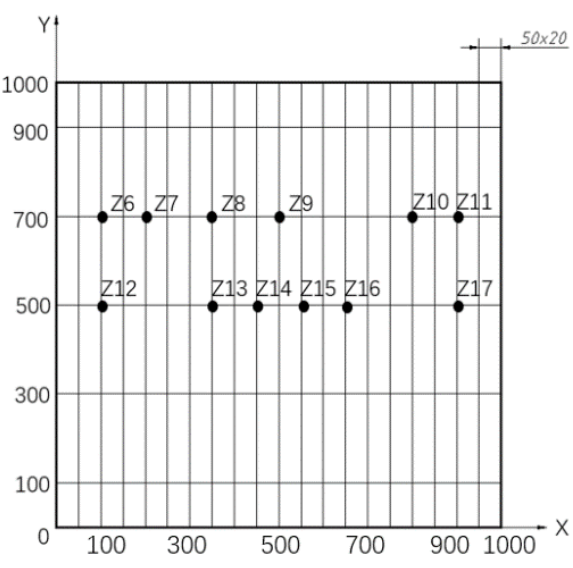

(b)

Fig.8 Strain point distribution chart of arbitrary point load

\subsection{Research on reconstruction of strain field}

Based on the information of the discrete point strain by experiments, it is necessary to obtain the strain field of the entire surface. More detailed strain information is obtained by interpolation of discrete point strains, and detailed strain information will be fitted to the strain cloud. In addition, strain field reconstruction can also serve as a cushion for deformation field reconstruction, providing direct input for deformation field reconstruction.

The inverse distance weighted averaging method is introduced below, which is an algorithm that supports the two-dimensional array interpolation, while the principle of this algorithm is shown in Fig.9.

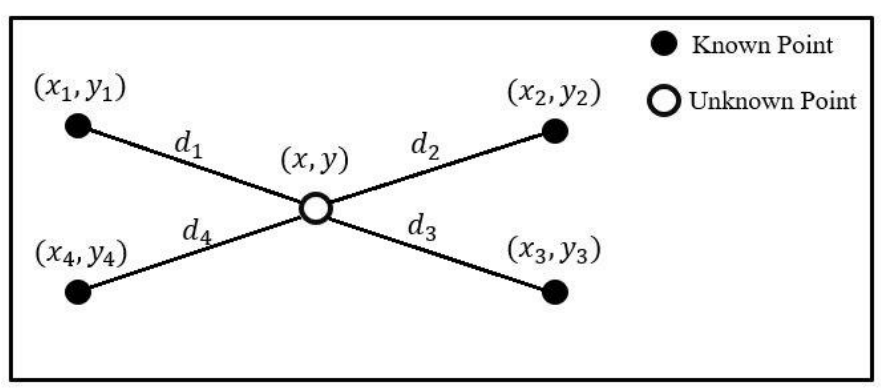

Fig.9 Schematic diagram for inverse distance weighted average method

(which algorithm?) This algorithm solves the value of an unknown point by weighting the distance from an unknown point to several known points around the same plane. The distances from unknown points to any known point are shown in Eq. (15).

$$
d_{k}=\sqrt{\left(x-x_{k}\right)^{2}+\left(y-y_{k}\right)^{2}}
$$

where, $d_{k}$ - The distance between unknown point and known point $k, \mathrm{~mm}$;

$x_{k}$ - The coordinate $\mathrm{x}$ of known point, $\mathrm{mm}$;

$y_{k}$ - The coordinate $\mathrm{y}$ of known point, $\mathrm{mm}$;

$x$ - The coordinate $\mathrm{x}$ of unknown point, $\mathrm{mm}$;

$y$ - The coordinate y of unknown point, $\mathrm{mm}$,

among which $k=1,2,3 \ldots n$. If the binary function $f(x, y)$ is the strain of an unknown point, $f(x, y)$ is expressed as Eq. (16):

$$
f(x, y)=\sum_{1}^{n} w_{k}(x, y) z_{k}
$$

The expression of $w_{k}(x, y)$ is Eq. (17): 


$$
w_{k}(x, y)=\frac{\frac{1}{d_{k}^{2}}}{\sum_{1}^{n} \frac{1}{d_{k}^{2}}}
$$

where, $z_{k}$ - The strain of known point $k$.

Reconstruction of strain field of thin plate is based on MATLAB by inverse distance weighted average method. Based on the principle of guaranteeing the integrity of reconstruction and minimizing the amount of computation, the interpolation dimension of reconstruction is chosen to be 300x20 after multiple reconstruction experiments in different dimensions. After the reconstruction of strain field, there are 300 strain data in direction $x$ and 20 strain data in direction $y$. In total there are 6000 strain data on the whole surface. In addition, the reconstructed $300 \times 20$ strain data are uniformly distributed on the thin plate surface. Fig.10 and Fig.11 show the reconstructed strain fields of the center point load and the arbitrary point load in different load values, respectively.

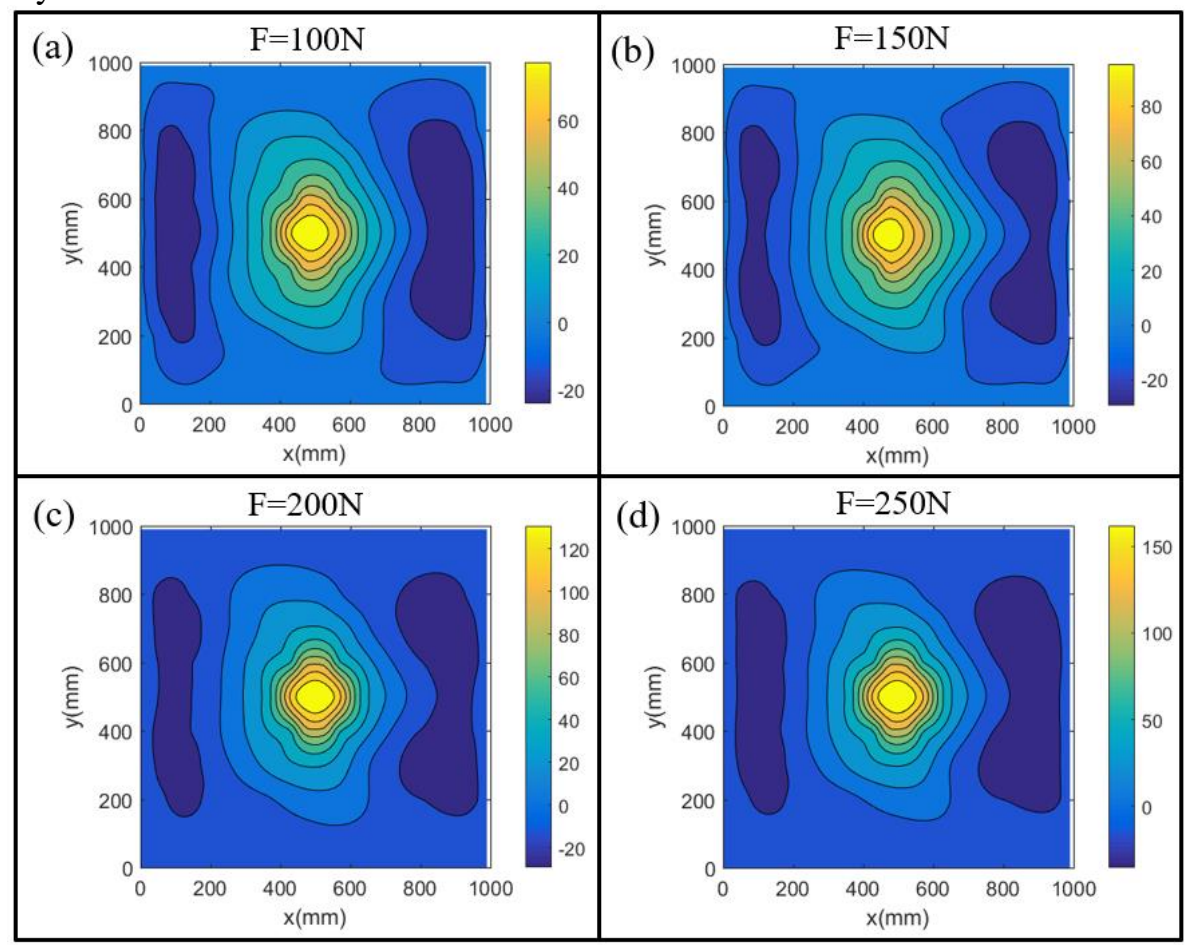

Fig.10 Strain cloud chart of center point load 


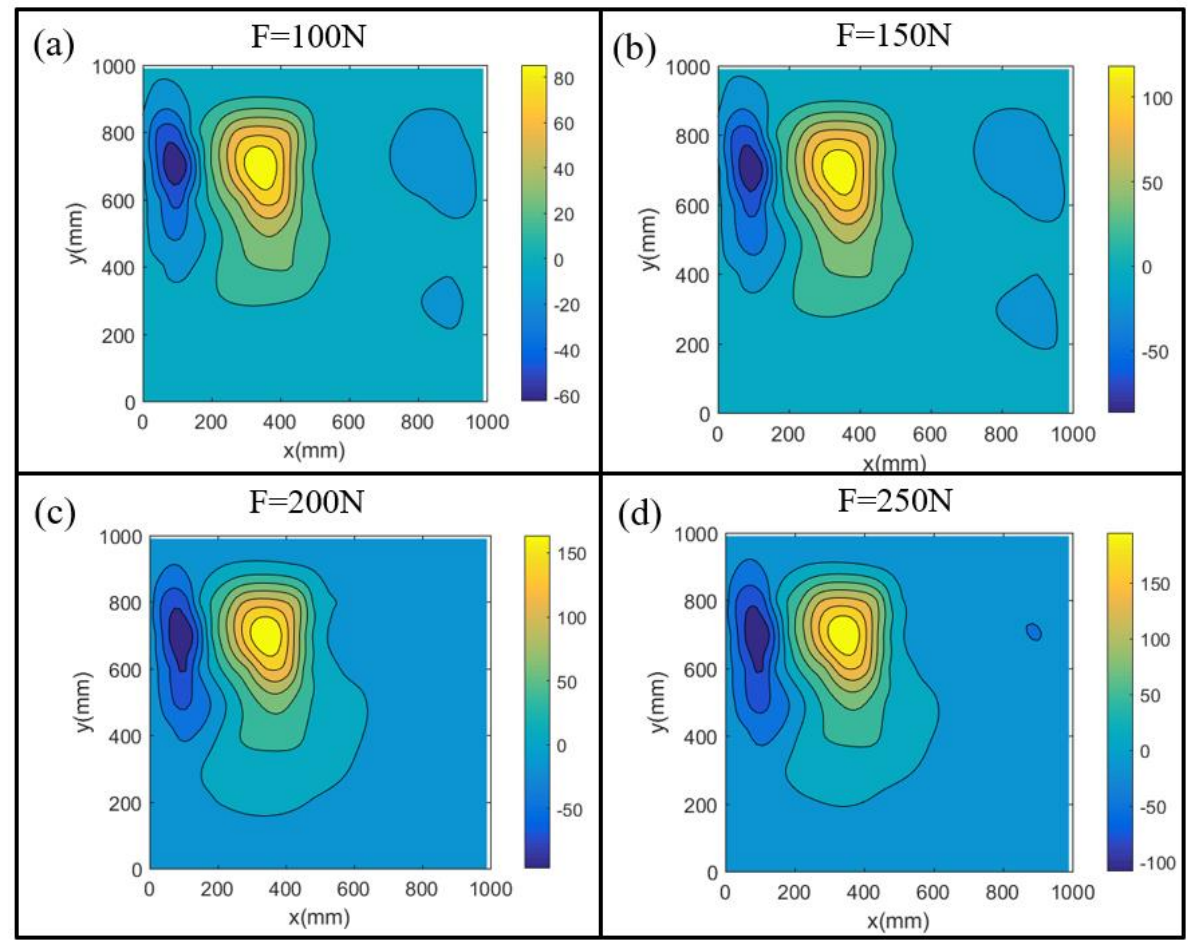

Fig.11 Strain cloud chart of arbitrary point load

\section{Reconstruction of deformation field and examples verification 4.1 Reconstruction algorithms of large deflection deformation field}

Based on the study of strain field reconstruction of thin plate, the results of strain field reconstruction are used to reconstruct the deformation field. According to the strain field, the strain is divided into 20 parallel lines along the y-axis, each of which contains 300 points of strain data along the $\mathrm{x}$-axis. When calculating the deformation, a straight line is taken as the research object, and the deformation of 300 points on this straight line is calculated by iteration. Then, the same method is used to calculate the residual linear deformation. Finally, the deformation data of 6000 points are fitted to the deformation surface of the whole thin plate, and the deformation field of the thin plate is obtained.

Taking a certain point as the research object, the deformation of the point is marked as $z_{i k}$, where, $i(i=1,2,3 \ldots 300)$ represents the serial number of the change point, and $k(k=1,2,3 \ldots 20)$ represents the serial number of the line where the point is located. According to the above conditions, rewrite Eq. (14) to Eq. (18):

$$
Z_{i k}=\iint \frac{\varepsilon_{x n m}^{-}(x)-\varepsilon_{x n m}^{+}(x)}{t} d x d x
$$

Because of the point-by-point iteration method, the quadratic integral expression Eq. (18) is transformed into the numerical integral expression Eq. (19):

$$
Z_{i k}=\sum_{n=1}^{i}\left(\sum_{n=1}^{i} \frac{a\left(\varepsilon_{x n m}^{-}-\varepsilon_{x n m}^{+}\right)}{t}\right)
$$

where, $a$ - Distance between two adjacent points on a straight line, $\mathrm{mm}$;

The length of a single line is $1000 \mathrm{~mm}$, which contains 300 data points. Therefore, we have $a=1000 \mathrm{~mm} / 300 \mathrm{~mm}=10 / 3$. The thickness of the thin plate $t=3 \mathrm{~mm}$ and the distance $a$ between adjacent points are introduced into formula Eq. (19), and the Eq. (20) is obtained. The analytical formula Eq. (20) for reconstruction of deformation field under large deflection which can be used for direct calculation:

$$
Z_{i k}=\sum_{n=1}^{i}\left(\sum_{n=1}^{i} \frac{10\left(\varepsilon_{x n m}^{-}-\varepsilon_{x n m}^{+}\right)}{9}\right)
$$

According to the above calculation method and analytical formula, the reconstructed flow 
chart of deformation field is drawn as Fig. 12.

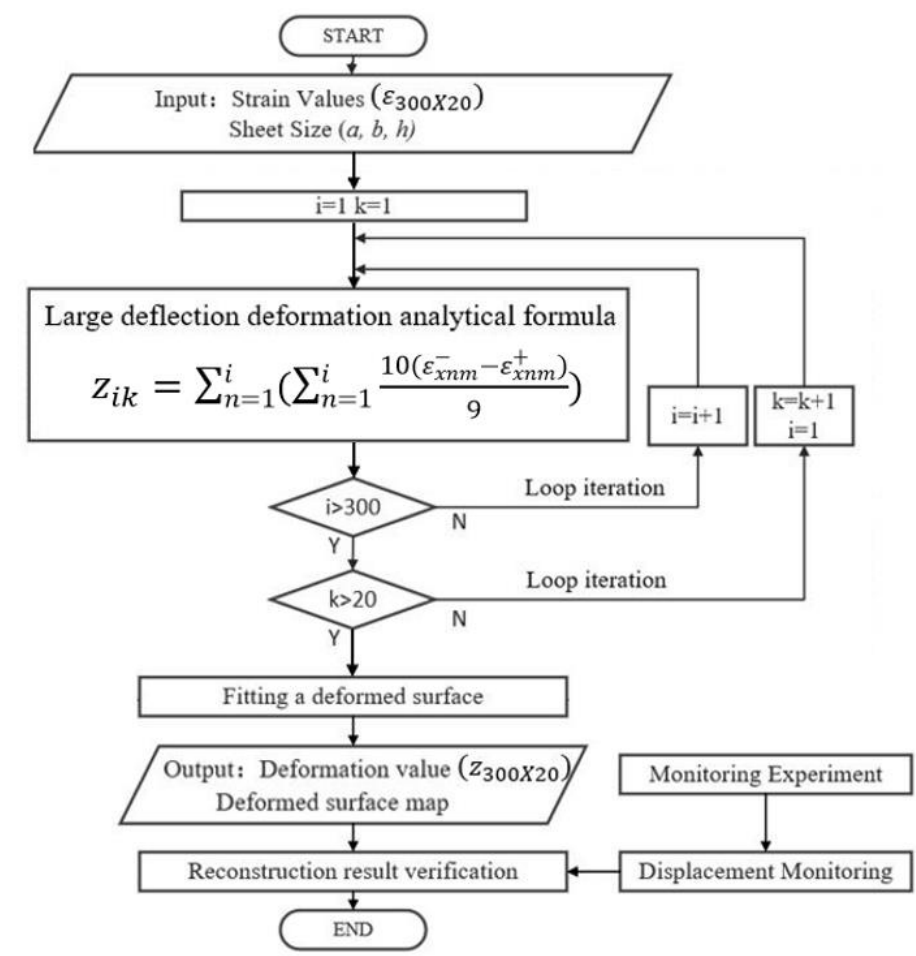

Fig.12 Flow chart of reconstruction algorithm for deformation field

As shown in Fig.12, the calculated input is strain data $\varepsilon_{300 \times 20}$ and thin plate size $a \times b \times h$, and the output is the deformation value $Z_{300 \times 20}$ corresponding to each strain data point, and the deformation surface of thin plate. In the calculation process, the final analytic formula Eq. (20) derived above is solved by the double cycle iteration method. The deformation corresponding to a strain data point is obtained by solving an analytic formula Eq. (20) once and recorded in the deformation matrix $Z_{300 \times 20}$ until all the points are solved.

\subsection{Reconstruction of deformation field and verification of examples}

Reconstruction of deformation field based on MATLAB by the 4.1 proposed method. Fig. 13 (a), (b), (c) and (d) show the deformed surface of the thin plate when the central point is loaded at $100 \mathrm{~N}, 150 \mathrm{~N}, 200 \mathrm{~N}$ and $250 \mathrm{~N}$. 


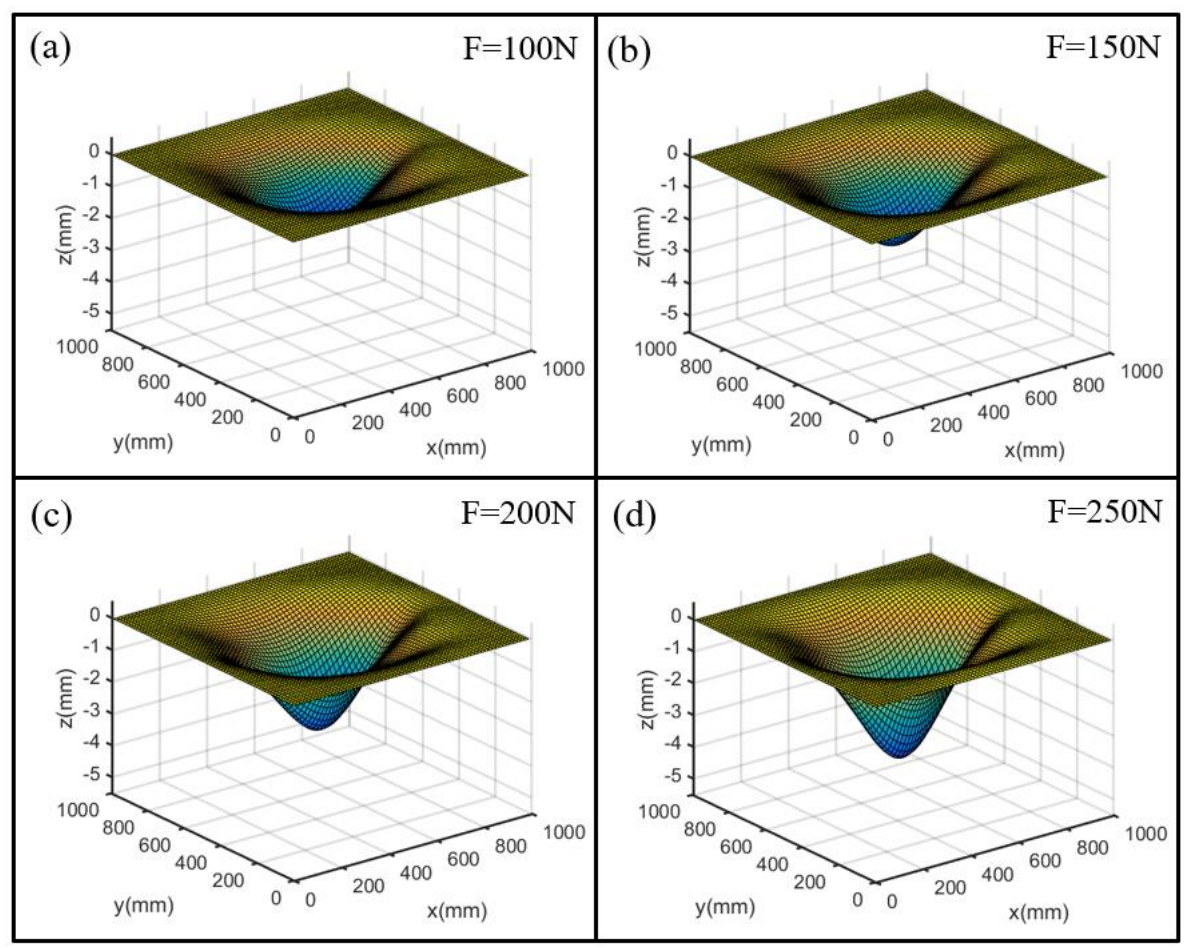

Fig.13 Deformation Surface Diagram under Central Point Loading

Tab.3 is a comparison of the reconstructed and experimental results of three displacement measuring points $\mathrm{A}, \mathrm{B}$ and $\mathrm{C}$ when the central point is loaded. It can be seen from the table that the average reconstruction error is $4.1 \%$ and the reconstruction error is less than $7 \%$. As can be observed from Tab.3, the average errors of points A, B and C are 4.2\%, 5.6\% and 2.9\% respectively. Point $\mathrm{C}$ has the smallest average error as the maximum deformation point (Loading point).

Tab.3 Comparisons between reconstructed results and experimental results under central point loading

\begin{tabular}{ccccc}
\hline $\begin{array}{c}\text { Monitoring } \\
\text { points }\end{array}$ & $\begin{array}{c}\text { Load value } \\
(\mathbf{N})\end{array}$ & $\begin{array}{c}\text { Experimental } \\
\text { result }(\mathbf{m m})\end{array}$ & $\begin{array}{c}\text { Refactoring } \\
\text { result (mm) }\end{array}$ & $\begin{array}{c}\text { Error } \\
(\mathbf{\%})\end{array}$ \\
\hline $\mathbf{A}$ & 100 & -1.11 & -1.04 & 6.3 \\
$\mathbf{A}$ & 150 & -1.52 & -1.44 & 5.2 \\
$\mathbf{A}$ & 200 & -1.89 & -1.80 & 4.8 \\
$\mathbf{A}$ & 250 & -2.22 & -2.12 & 4.5 \\
\hline $\mathbf{B}$ & 100 & -1.44 & -1.34 & 6.8 \\
$\mathbf{B}$ & 150 & -1.97 & -1.85 & 6.1 \\
$\mathbf{B}$ & 200 & -2.44 & -2.31 & 5.3 \\
$\mathbf{B}$ & 250 & -2.86 & -2.74 & 4.2 \\
\hline $\mathbf{C}$ & 100 & -2.15 & -2.07 & 3.7 \\
$\mathbf{C}$ & 150 & -2.52 & -2.44 & 3.2 \\
$\mathbf{C}$ & 200 & -3.61 & -3.51 & 2.7 \\
$\mathbf{C}$ & 250 & -4.21 & -4.11 & 2.3 \\
\hline
\end{tabular}

Fig. 14 (a), (b), (c) and (d) show the deformed surface of the thin plate when the arbitrary point is loaded at $100 \mathrm{~N}, 150 \mathrm{~N}, 200 \mathrm{~N}$ and $250 \mathrm{~N}$. 


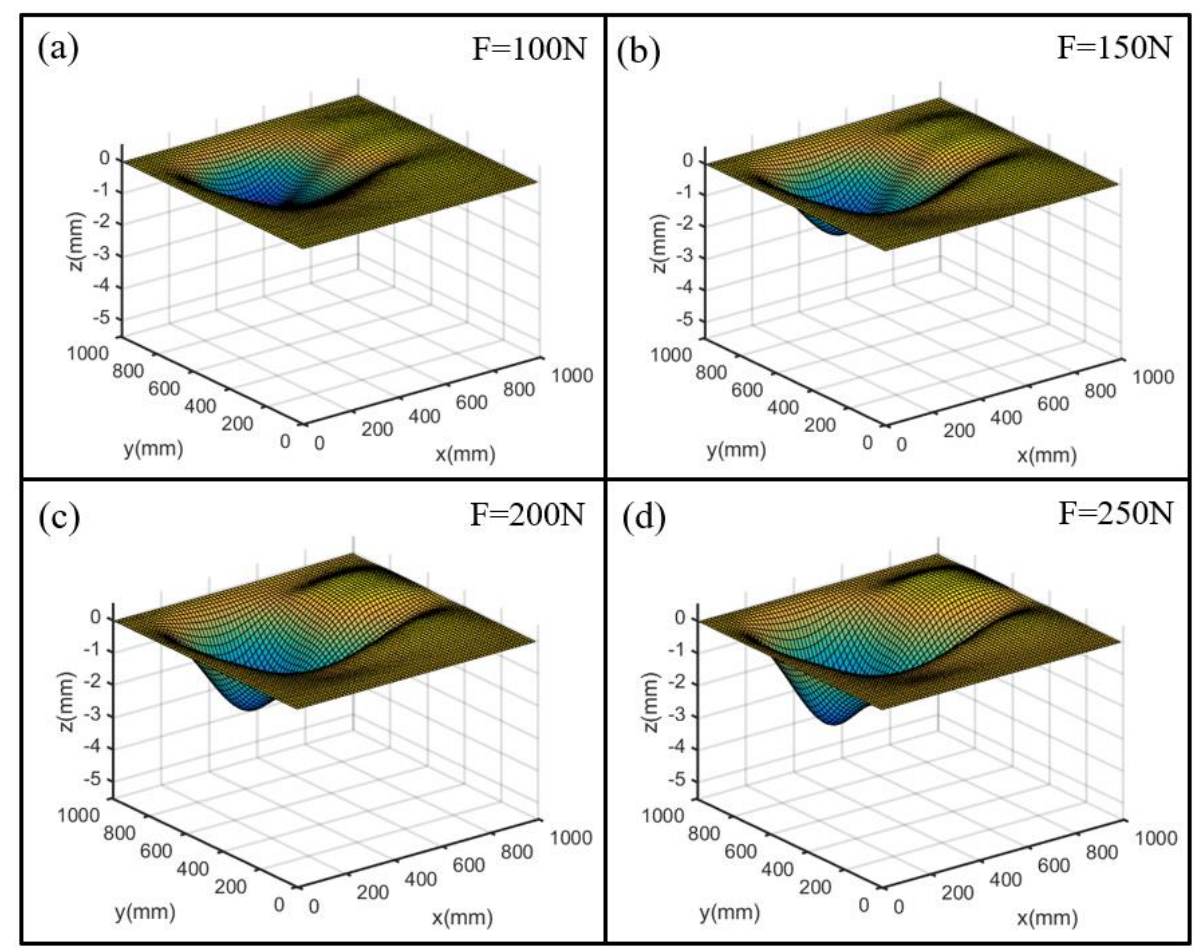

Fig.14 Deformation Surface Diagram under Arbitrary Point Loading

Tab.4 is a comparison of the reconstructed and experimental results of three displacement measuring points $\mathrm{A}, \mathrm{B}$ and $\mathrm{C}$ when the arbitrary point is loaded. It can be seen from the table that the average reconstruction error is $5.8 \%$ and the reconstruction error is less than $9 \%$. As can be seen from Tab.4, the average errors of points A, B and C are 3.5\%, $6.9 \%$ and $6.8 \%$, and point $\mathrm{A}$ as the maximum deformation point (Loading point) has the smallest average error.

Tab.4 Comparisons between reconstructed results and experimental results under arbitrary point loading

\begin{tabular}{ccccc}
\hline $\begin{array}{c}\text { Monitoring } \\
\text { points }\end{array}$ & $\begin{array}{c}\text { Load value } \\
\mathbf{( N )}\end{array}$ & $\begin{array}{c}\text { Experimental } \\
\text { result }(\mathbf{m m})\end{array}$ & $\begin{array}{c}\text { Refactoring } \\
\text { result }(\mathbf{m m})\end{array}$ & $\begin{array}{c}\text { Error } \\
(\mathbf{\%})\end{array}$ \\
\hline $\mathbf{A}$ & 100 & -1.61 & -1.54 & 4.3 \\
$\mathbf{A}$ & 150 & -2.28 & -2.19 & 3.9 \\
$\mathbf{A}$ & 200 & -2.87 & -2.78 & 3.1 \\
$\mathbf{A}$ & 250 & -3.38 & -3.29 & 2.7 \\
\hline $\mathbf{B}$ & 100 & -1.22 & -1.12 & 8.2 \\
$\mathbf{B}$ & 150 & -1.73 & -1.61 & 7.0 \\
$\mathbf{B}$ & 200 & -2.16 & -2.02 & 6.5 \\
$\mathbf{B}$ & 250 & -2.57 & -2.41 & 6.2 \\
\hline $\mathbf{C}$ & 100 & -1.13 & -1.04 & 7.9 \\
$\mathbf{C}$ & 150 & -1.58 & -1.46 & 6.9 \\
$\mathbf{C}$ & 200 & -1.98 & -1.85 & 6.6 \\
$\mathbf{C}$ & 250 & -2.33 & -2.19 & 6.0 \\
\hline
\end{tabular}

According to the comparison between the results of deformation reconstruction and experimental results, the reconstruction error of center point loading $(4.1 \%)$ is smaller than that of arbitrary point loading $(5.8 \%)$. In addition, the reconstruction error of the maximum deformation point (Loading point) (2.7\%/3.5\% in center/arbitrary point loading) is smaller than that of the other two points (4.9\%/6.9\% in center/arbitrary point loading).

\section{Conclusion}

This paper proposes a large deflection deformation reconstruction method of elastic thin plate based on strain monitoring. The main contributions are summarised as follows:

(1) According to the large deflection deformation hypothesis, the theoretical analysis of the 
relationship between strain and deformation of thin plate was completed. Based on obtaining the deformation expression from strain information, a method (what method?) was proposed for solving the expression and get the analytical solution of deformation.

(2) Construction of strain monitoring experimental platform. The layout scheme of test strain measuring points and the loading conditions of the experiment were identified. Strain monitoring experiment on thin plate was carried out, and the strain field of the entire thin plates surface based on the result of experiments was obtained.

(3) The specific size and strain field of thin plate are brought into the analytical expression of deformation. The method of calculation and the flow chart of the algorithm was designed. Finally, the deformed surfaces of thin plates under different working conditions were obtained. The reconstruction error under center point loading and arbitrary point loading conditions was within 7\% and $9 \%$, and the reconstruction of the deformation field is completed.

Based on the strain measured by experiments, the deformation field were solved by analytical solution based on theory. The accuracy of analytical solutions was verified in experiments. The reconstructed results showed that this method is applicable to the monitoring of large deflection deformation field of thin elastic plates with four fixed side. (who provides ideas? What ideas? 'idea' is a very informal word in scientific articles!) also provides ideas for deformation monitoring of thin plate structures.

\section{Acknowledgement:}

This work was supported by the Fundamental Research Funds for the Central Universities (No. FRF-GF-17-B21).

\section{References:}

[1] Martin H. Sadd, ElasticityTheory, Applications and Numerics, Elsevier Inc, 2005: 230-235.

[2] Tessler A, Spangler J L, A variational principle for reconstruction of elastic deformations in shear deformable plates and shells, NASATM-2003-212445. 2003.

[3] Tessler A, Spangler J L, Inverse FEM for full-field reconstruction of elastic deformations in shear deformable plates and shells, Proceedings of 2nd European Work-shop on Structural Health Monitoring, Munich, Germany. 2004.

[4] Tessler A, Spangler J L, Gherlone M, Deformed shape and stress reconstruction in plate and shell structures undergoing large displacements: application of inverse finite element method using fiber bragg grating strains, Proceedings of 10th World Congress on Computational Mechanics, Sao Paulo, Brazil. 2012.

[5] Kefal A, Oterkus E, Displacement and stress monitoring of a chemical tanker based on inverse finite element method, Ocean Engineering, 2016, 112: 33-46.

[6] Kefal A, Mayang J B, Oterkus E, Three dimensional shape and stress monitoring of bulk carriers based on iFEM methodology, Ocean Engineering, 2018, 147: 256-267.

[7] Cerracchio, P. Gherlone, M. DiSciuva, M. Tessler, Shape and Stress Sensing of Multilayered Composite and Sandwich Structures Using an Inverse Finite Element Method, Proceedings of V International Conference on Computational Methods for Coupled Problems in Science and Engineering, Ibiza, Spain. 2013.

[8] Cerracchio P, Gherlone M, Di Sciuva M, A novel approach for displacement and stress monitoring of sandwich structures based on the inverse finite element method, Composite Structures, 2015, 127: 69-76.

[9] Kefal A, Structural health monitoring of marine structures by using inverse finite element method, University of Strathclyde, 2017.

[10] Kefal A, Tessler A, Oterkus E, An enhanced inverse finite element method for displacement and stress monitoring of multilayered composite and sandwich structures, Composite Structures, 2017, 179: 514-540.

[11] Kim H-I, Kang L-H, Han J-H, Shape estimation with distributed fiber Bragg grating sensors for rotating structures, Smart Materials and Structures, 2011, 20(3): 035011.

[12] Davis M, Kersey A, Sirkis J, Shape and vibration mode sensing using a fiber optic Bragg grating array, Smart Materials and Structures, 1996, 5(6): 759 
[13] Li C-J, Ulsoy A G, High-precision measurement of tool-tip displacement using strain gauges in precision flexible line boring, Mechanical Systems and Signal Processing, 1999, 13(4): 531546

[14] Li C-J, Ulsoy A G, Endres W J, The Effect of Tool Rotation on Regenerative Chatter in Line Boring. Dynamics, Acoustics and simulations, ASME publication DE, 1998, 98: 235-243

[15] Stöbener U, Gaul L, Modal vibration control for PVDF coated plates, Journal of Intelligent Material Systems and Structures, 2000, 11(4): 283-293

[16] Ko W L, Fleischer V T, Improved displacement transfer functions for structure deformed shape predictions using discretely distributed surface strains, NASA/TP-2012-216060. 2012.

[17] Nicolas M J, Structural analysis and testing of a carbon-composite wing using fiber Bragg gratings, Mississippi State University, 2013.

[18] Derkevorkian A, Masri S F, Alvarenga J, Strain-based deformation shape-estimation algorithm for control and monitoring applications, AIAA journal, 2013, 51(9): 2231-2240.

[19] Yi J, Zhu X, Zhang H, Spatial shape reconstruction using orthogonal fiber Bragg grating sensor array, Mechatronics, 2012, 22(6): 679-687.

[20] Guo Meng, He Hanhui, Xiao Dingbang, Satellite antenna array deformation detection based on strain measurement method, Spacecraft Environment Engineering, 2012, 29(6): 663-666(in Chinese.

[21] J. Liu, W.D. Zhu, P.G. Charalambides, Y.M. Shao, Y.F. Xu, X.M. Fang, A dynamic model of a cantilever beam with a closed, embedded horizontal crack including local flexibilities at crack tips, Journal of Sound and Vibration, 2016, 382:274-290.

[22] E Reissner, On the theory of bending of elastic plates, J.math.phys, 23(1944): 184-191.

[23] Tessler A, Spangler J L, A least-squares variational method for full-field reconstruction of elastic deformations in shear-deformable plates and shells, Computer methods in applied mechanics and engineering, 2005, 194(2-5): 327-339. 